\title{
Soret-Dufour Effects on the MHD Flow and Heat Transfer of Microrotation Fluid over a Nonlinear Stretching Plate in the Presence of Suction
}

\author{
Md Abdullah Al Mahbub ${ }^{1}$, Nasrin Jahan Nasu ${ }^{2}$, Shomi Aktar ${ }^{1}$, Zillur Rahman ${ }^{1}$ \\ ${ }^{1}$ Department of Mathematics, Comilla University, Comilla, Bangladesh \\ ${ }^{2}$ Department of Accounting and Information System, University of Chittagong, Chittagong, Bangladesh \\ Email: dipmahbub13@yahoo.com
}

Received March 28, 2013; revised April 29, 2013; accepted May 4, 2013

Copyright (C) 2013 Md Abdullah Al Mahbub et al. This is an open access article distributed under the Creative Commons Attribution License, which permits unrestricted use, distribution, and reproduction in any medium, provided the original work is properly cited.

\begin{abstract}
In this work, the Micropolar fluid flow and heat and mass transfer past a horizontal nonlinear stretching sheet through porous medium is studied including the Soret-Dufour effect in the presence of suction. A uniform magnetic field is applied transversely to the direction of the flow. The governing differential equations of the problem have been transformed into a system of non-dimensional differential equations which are solved numerically by Nachtsheim-Swigert iteration technique along with the sixth order Runge-Kutta integration scheme. The velocity, microrotation, temperature and concentration profiles are presented for different parameters. The present problem finds significant applications in hydromagnetic control of conducting polymeric sheets, magnetic materials processing, etc.
\end{abstract}

Keywords: Heat Transfer; Micropolar Fluid; Porous Media; Stretching Sheet; Soret Number; Dufour Number

\section{Introduction}

The natural convection processes involving the combined mechanism of heat and mass transfer are encountered in many natural and industrial transport processes such as hot rolling, wire drawing, spinning of filaments, metal extrusion, crystal growing, continuous casting, glass fiber production, paper production, and polymer processing, etc. Ostrach [1] the initiator of the study of convection flow, made a technical note on the similarity solution of transient free convection flow past a semi infinite vertical plate by an integral method. Goody [2] considered a neutral fluid. Sakiadis [3] analyzed the boundary layer flow over a solid surface moving with a constant velocity. This boundary layer flow situation is quite different from the classical Blasiuss problem of boundary flow over a semi-infinite flat plate due to entrainment of ambient fluid.

Micropolar fluids, distinctly non-Newtonian in nature, are referred to those that contain micro-constituents belonging to a class of complex fluids with nonsymmetrical stress tensor. These fluids respond to micro-rotational motions and spin inertia, and therefore can support couple stress and distributed body torque which are not achievable with the classical Navier-Stokes equations or the viscoelastic flow models. The Micropolar fluid models are developed to make an analysis of the flow characteristics of physiological fluids (blood containing corpuscles), colloidal suspensions, paints, liquid crystal suspensions, concentrated silica particle suspensions, oils containing very fine suspensions, industrial contaminants containing toxic chemicals, lubricants, organic/inorganic hybrid nano-composites and clay which are fabricated by melt intercalation etc. Eringen [4] first designed the study on micropolar fluid making an analysis on the theory of micropolar fluids which provided a mathematical model for non-Newtonian behavior.

Crane [5] noted that usually the sheet is assumed to be inextensible, but situations may arise in the polymer industry in which it is necessary to deal with a stretching plastic sheet. For examples, materials manufactured by aerodynamic extrusion processes and heat-treated materials traveling between a feed roll and a wind-up roll or on a conveyor belt possess the characteristics of a moving continuous stretching surface. Moreover lots of metallurgical processes occupy the system of cooling of continuous strips or filaments by drawing them through a quiescent fluid and that in the process of drawing, these strips are sometimes stretched. 
An important matter is that the final product depends to a great extent on the rate of cooling. By drawing such strips in an electrically conducting fluid subjected to a magnetic field, the rate of cooling can be controlled and a final product of desired characteristics can be achieved. The study of heat and mass transfer is necessary for determining the quality of the final product. Sparrow [6] explained a parameter named Rosseland approximation to describe the radiation heat flux in the energy equation in his book.

The boundary layer models for steady or unsteady micropolar fluids in various geometries (stationary or moving surface, linear or nonlinear stretching surface etc.) with/or without heat transfer considering various flow conditions (no slip or slip, suction/injection at the surface) and thermal boundary conditions (constant/variable surface temperature or heat flux) have extensively been studied by numerous researchers [7-16].

Moreover, the thermal-diffusion (Soret) effect, for instance, has been utilized for isotope separation, and in mixtures between gases with very light molecular weight $(\mathrm{Hz}, \mathrm{He})$ and of medium molecular weight $(\mathrm{Nz}$, air) the diffusion-thermo (Dufour) effect was found to be of a considerable magnitude such that it cannot be ignored, described by Eckert and Drake [17] in their book. Recently plenty of investigators [18-21] are getting interest work on Soret-Dufour effects.

From the above referenced work and the numerous possible industrial applications of the problem, it is of paramount interest in this study in order to clarify the parametric behavior of magneto-hydrodynamic flow of free convection of a micropolar fluid over a nonlinear stretching sheet in the presence of dynamic effects of suction, thermal-diffusion and diffusion-thermo.

\section{Mathematical Model}

We consider the isothermal, steady, laminar, hydromagnetic free convection flow of an incompressible micropolar fluid flowing past a nonlinear stretching sheet coinciding with the plane $y=0$, the flow being confined in the region $y>0$. The flow under consideration is also subjected to a strong transverse magnetic field $B_{0}$ with a constant intensity along the $y$-axis.

Two equal and opposite forces are introduced along the $x$-axis so that the surface is stretched keeping the origin fixed. The flow configurations and the coordinate system are shown in Figure 1. We assume that the velocity of a point on a sheet is proportional to its distance from the slit. We assume that all the fluid properties are isotropic and constant. Under the usual boundary layer and Boussinesq approximations, the governing equations for the problem under consideration can be written as follows:

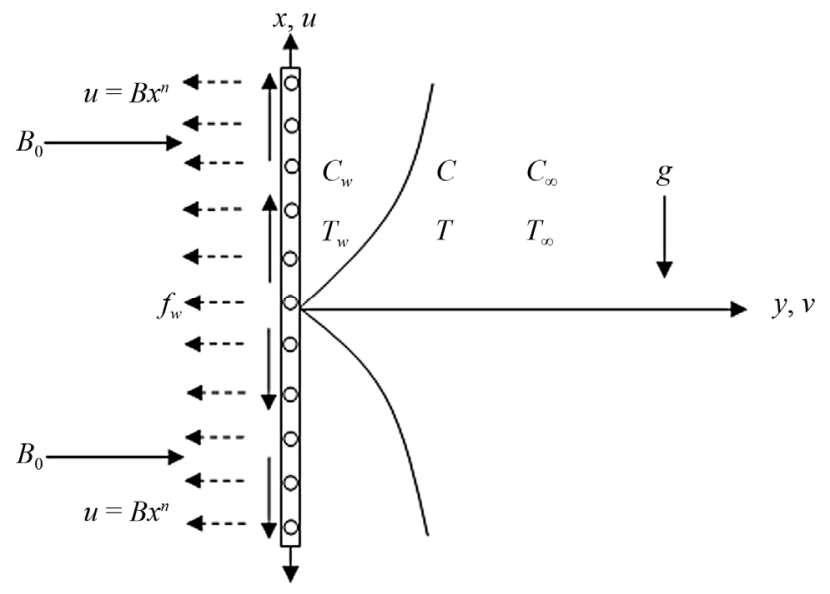

Figure 1. Flow configuration and coordinate system.

$$
\frac{\partial u}{\partial x}+\frac{\partial v}{\partial y}=0
$$

$$
\begin{gathered}
u \frac{\partial u}{\partial x}+v \frac{\partial u}{\partial y} \\
=\left(v+\frac{S}{\rho}\right) \frac{\partial^{2} u}{\partial y^{2}}+g \beta\left(T-T_{\infty}\right)-\frac{\sigma B_{0}^{2}}{\rho} u+\frac{S}{\rho} \frac{\partial N}{\partial y}-\frac{\left(v+\frac{S}{\rho}\right)}{k_{p}} u \\
u \frac{\partial N}{\partial x}+v \frac{\partial N}{\partial y}=\frac{v_{S}}{\rho j} \frac{\partial^{2} N}{\partial y^{2}}-\frac{S}{\rho j}\left(2 N+\frac{\partial u}{\partial y}\right) \\
u \frac{\partial T}{\partial x}+v \frac{\partial T}{\partial y} \\
=\frac{\kappa}{\rho c_{p}} \frac{\partial^{2} T}{\partial y^{2}}+\frac{\left(v+\frac{S}{\rho}\right)}{c_{p}}\left[\frac{u^{2}}{k}+\left(\frac{\partial u}{\partial y}\right)^{2}\right]+\frac{D_{m} K_{T}}{c_{s} c_{p}} \frac{\partial^{2} C}{\partial C^{2}} \\
u \frac{\partial C}{\partial x}+v \frac{\partial C}{\partial y}=D_{m} \frac{\partial^{2} C}{\partial y^{2}}+\frac{D_{m} K_{T}}{T_{m}} \frac{\partial^{2} T}{\partial y^{2}}
\end{gathered}
$$

In Equation (2) the Darcian porous drag force term is defined by the term, $-\left(v+\frac{S}{\rho}\right) \frac{u}{k_{p}}$, which is linear in terms of the translational velocity, $u$. With $S=0$, the micropolar effects disappears and this term reduces to the conventional Newtonian Darcy drag force i.e. $-v \frac{u}{k_{p}}$. The micro-rotation component, $N$, is coupled to the linear momentum Equation (2) via the angular velocity gradient term, $\frac{S}{\rho} \frac{\partial N}{\partial y}$. Very strong coupling exists between the translational velocity components, $u$ and $v$, in Equation (3) via the convective acceleration terms, $u \frac{\partial N}{\partial x}$ and 
$v \frac{\partial N}{\partial y}$. Furthermore, there is a second coupling term linking the angular velocity with the $x$-direction velocity gradient, in Equation (3), $-\frac{S}{\rho j}\left(2 N+\frac{\partial u}{\partial y}\right)$. The microrotation viscosity (or spin-gradient viscosity) $v_{S}$ is defined by $v_{S}=\left(\mu+\frac{S}{2}\right) j \quad$ (Rahman [15]). We note that in the viscous shear diffusion term, $\left(v+\frac{S}{\rho}\right) \frac{\partial^{2} u}{\partial y^{2}}$, the Newtonian kinematic viscosity is now supplemented by the Eringen micropolar vortex viscosity, $S$. In the present work, we assume that the micro-inertia per unit mass $j$ is a constant. Also, positive or negative $n$ indicate the acceleration and deceleration of the sheet from the extruded slit respectively. Here $(u, v)$ are the fluid velocity components in the $x$-, $y$-directions respectively, $N$ is the microrotation, $T$ is the temperature, $v$ is the kinematic viscosity, $\rho$ is the fluid density, $\sigma$ is the electric conductivity, $g$ is the acceleration due to gravity, $\beta$ is the volumetric coefficient of thermal expansion, $B_{0}$ is the uniform magnetic field strength, $k_{p}$ is the Darcy permeability of porous medium, $j$ is the Microinertia per unit mass, $\kappa$ is the thermal conductivity of the fluid, $c_{p}$ the specific heat at constant pressure, $D_{m}$ is the chemical molecular diffusivity, $K_{T}$ is the Thermophoretic constant, $T_{m}$ is the Mean fluid temperature and $c_{s}$ is the Concentration susceptibility.

The appropriate boundary conditions suggested by the physical conditions are:

1) on the plate surface at $y=0$ :

$$
\begin{aligned}
& u=U_{w}=B x^{n}, v=v_{0}(x), N=-S \frac{\partial u}{\partial y}, \\
& T=T_{w}\left(=T_{\infty}+A x^{\gamma}\right), C=C_{w}\left(=C_{\infty}+D x^{\gamma}\right)
\end{aligned}
$$

2) matching with the quiescent free stream as $y \rightarrow \infty$ :

$$
u \rightarrow U_{\infty}, N \rightarrow 0, T \rightarrow T_{\infty}, C \rightarrow C_{\infty}
$$

where the subscripts $w$ and $\infty$ refer to the wall and boundary layer edge, respectively. The relationship between the microrotation function $N$ and the surface shear $\frac{\partial u}{\partial y}$ is chosen for investigating the effect of different surface conditions for the microrotation of the micropolar fluid elements. The conditions are generally of importance in micropolar boundary layer analysis. When microrotation parameter $S=0$, we obtain $N=0$ which represents no-spin condition i.e. the microelements in a concentrated particle flow-close to the wall are not able to rotate (Rahman [15]). Finally $A, D$ and $\gamma$ are the constants.

\subsection{Similarity Solutions}

The partial differential Equations (1) to (5) are transformed into non-dimensional form by mean of following dimensionless variables

$$
\left.\begin{array}{l}
\eta=y\left[\frac{B(n+1)}{2 v}\right]^{\frac{1}{2}} x^{\frac{n-1}{2}}, u=B x^{n} f^{\prime}(\eta), \\
v=-\left[B v\left(\frac{n+1}{2}\right)\right]^{\frac{1}{2}} x^{\frac{n-1}{2}}\left[f+\frac{n-1}{n+1} \eta f^{\prime}\right], \\
N=B\left[\frac{B(n+1)}{2 v}\right]^{\frac{1}{2}} x^{\frac{3 n-1}{2}} h(\eta), \theta(\eta)=\frac{T-T_{\infty}}{T_{w}-T_{\infty}}, \\
\phi(\eta)=\frac{C-C_{\infty}}{C_{w}-C_{\infty}}
\end{array}\right\}
$$

Implementing Equation (8) into Equations (1) to (5) produces the following ordinary differential equations:

$$
\begin{gathered}
(1+\Delta) f^{\prime \prime \prime}+f^{\prime \prime} f-\frac{2}{(n+1)}\left(M+\frac{(1+\Delta)}{D a}\right) f^{\prime} \\
-\frac{2}{(n+1)} n f^{\prime 2}+\Delta h^{\prime}+\frac{2}{(n+1)} G r \theta=0 \\
\left(1+\frac{\Delta}{2}\right) h^{\prime \prime}-\frac{2}{(n+1)} \Delta \frac{1}{\xi}\left(2 h+f^{\prime \prime}\right)-\frac{3 n-1}{n+1} h f^{\prime}+h^{\prime} f=0 \\
\frac{1}{\operatorname{Pr}} \theta^{\prime \prime}+\theta^{\prime} f-\frac{2}{n+1} \gamma \theta f^{\prime} \\
+(1+\Delta) E c\left[f^{\prime \prime 2}+\frac{2}{(n+1)} \frac{1}{D a} f^{\prime 2}\right]+D u \phi^{\prime \prime}=0 \\
\phi^{\prime \prime}+S c \phi^{\prime} f-\frac{2}{(n+1)} S c \gamma \phi f^{\prime}+S c S r \theta^{\prime \prime}=0
\end{gathered}
$$

and corresponding boundary conditions are reduce to:

$$
\left.\begin{array}{l}
f=f_{w}, f^{\prime}=1, h=-S f^{\prime \prime}, \theta=1, \phi=1 \text { at } \eta=0 \\
f^{\prime} \rightarrow 0, h \rightarrow 0, \theta \rightarrow 0, \phi \rightarrow 0 \text { as } \eta \rightarrow \infty
\end{array}\right\}
$$

where the primes denote differentiation with respect to $\eta$ (non-dimensional $y$-coordinate) and $\Delta=\frac{S}{\mu}$ is the vortex viscosity parameter, $G r=\frac{g \beta\left(T_{w}-T_{\infty}\right) x}{U_{w}^{2}}$ is the local Grashof number, $M=\frac{\sigma B_{0}^{2} x}{\rho U_{w}}$ is the local magnetic parameter and $B_{0}=\frac{B}{\sqrt{x}}$ is the magnetic field, $D a=\frac{k_{p}}{x^{2}}$ 
is the Darcy number, $\xi=\frac{j U_{w}}{v x}$ is the micro-inertia density parameter, $\operatorname{Pr}=\frac{\rho v c_{p}}{\kappa}$ is the Prandtl number, $E c=\frac{U_{w}^{2}}{c_{p}\left(T_{w}-T_{\infty}\right)}$ is the Eckert number, $D u=\frac{D_{m} K_{T}\left(C_{w}-C_{\infty}\right)}{v c_{s} c_{p}\left(T_{w}-T_{\infty}\right)}$ is the Dufour number, $S c=\frac{v}{D_{m}}$ is the Schmidt number, $S r=\frac{D_{m} K_{T}\left(T_{w}-T_{\infty}\right)}{T_{m}\left(C_{w}-C_{\infty}\right)}$ is the Soret number and $f_{w}=-v_{0}(x)\left[B v\left(\frac{n+1}{2}\right)\right]^{-\frac{1}{2}} x^{-\frac{n-1}{2}}$ is the suction parameter.

\subsection{Skin Friction, Rate of Heat and Mass Transfer}

The parameters of engineering interest for the present problem are the skin friction coefficient $\left(c_{f}\right)$, plate couple stress $\left(M_{w}\right)$, local Nusselt number $(N u)$ and Sherwood number $(S h)$ which indicate physically the wall shear stress, couple stress, the rate of heat transfer and the local surface mass flux respectively. The dimensionless skin-friction coefficient, Couple stress, Nusselt number and Sherwood number for impulsively started plate are given by

$$
\begin{gathered}
C_{f}=\frac{\tau_{w}}{\frac{1}{2} \rho U^{2}}=2 \sqrt{\frac{(n+1)}{2 \operatorname{Re}}} f^{\prime \prime}(0) \\
M_{w}=\frac{j\left(\mu+\frac{S}{2}\right)}{\frac{1}{2} \rho v U_{w}}\left(\frac{\partial N}{\partial y}\right)_{y=0}=\left(1+\frac{\Delta}{2}\right) \xi(n+1) h^{\prime}(0) \\
N u=-\left.\frac{x}{\left(T_{w}-T_{\infty}\right)} \frac{\partial T}{\partial y}\right|_{y=0}=-\sqrt{\frac{\operatorname{Re}(n+1)}{2}} \theta^{\prime}(0) \\
S h=\left.\frac{x}{\left(C_{w}-C_{\infty}\right)} \frac{\partial C}{\partial y}\right|_{y=0}=-\sqrt{\frac{\operatorname{Re}(n+1)}{2}} \phi^{\prime}(0)
\end{gathered}
$$

where $\mathrm{Re}$ is the Reynolds number. And hence the values proportional to the skin-friction coefficient, couple stress, Nusselt number and Sherwood number are $f^{\prime \prime}(0), h^{\prime}(0),-\theta^{\prime}(0)$ and $-\phi^{\prime}(0)$ respectively.

\section{Numerical Computation}

The numerical solutions of the non-linear differential Equations (9) to (12) under the boundary conditions (13) have been performed by applying a shooting method namely Nachtsheim and Swigert [22] iteration technique (guessing the missing values) along with sixth order Runge-Kutta iteration scheme. We have chosen a step size $\Delta \eta=0.01$ to satisfy the convergence criterion of $10^{-6}$ in all cases. The value of $\eta_{\infty}$ has been found to each iteration loop by $\eta_{\infty}=\eta_{\infty}+\Delta \eta$. The maximum value of $\eta_{\infty}$ to each group of parameters $\Delta, n, M, G r$, $D a, \xi, \operatorname{Pr}, E c, S, S c, \gamma, D u, S r$ and $f_{w}$ has been determined when the values of the unknown boundary conditions at $\eta=0$ not change to successful loop with error less than $10^{-6}$. In order to verify the effects of the step size $\Delta \eta$, we have run the code for our model with three different step sizes as $\Delta \eta=0.01, \Delta \eta=0.005$ and $\Delta \boldsymbol{\eta}=$ 0.001, and in each case we have found excellent agreement among them shown in Figures 2-5.

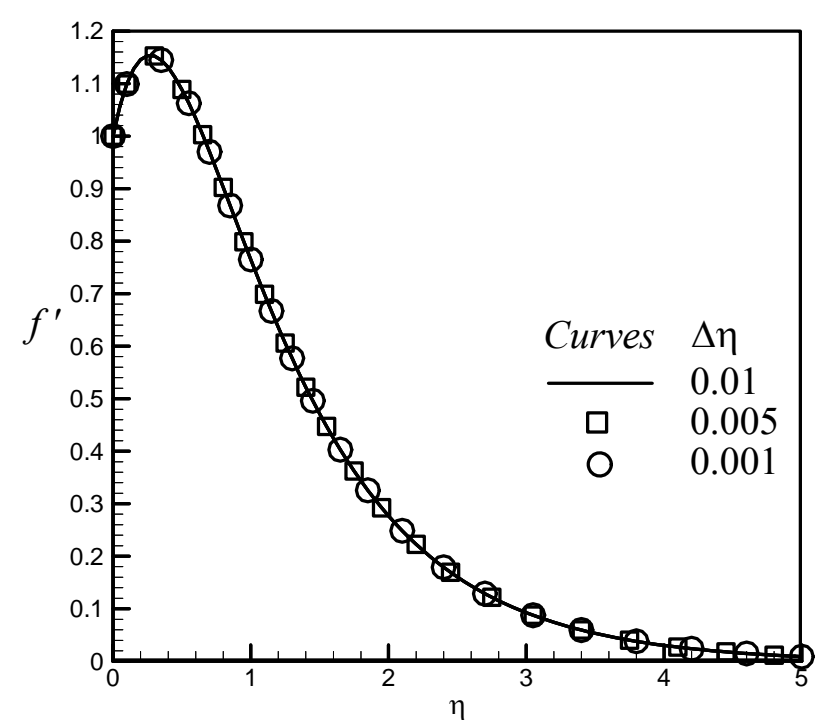

Figure 2. Distribution of velocity profiles for $\Delta \eta$.

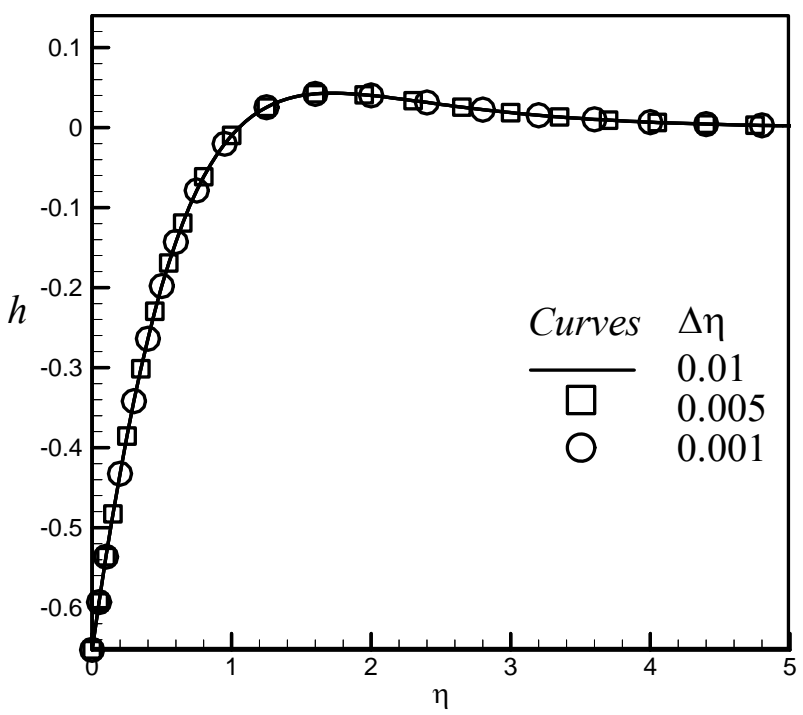

Figure 3. Distribution of microrotation profiles for $\Delta \eta$. 


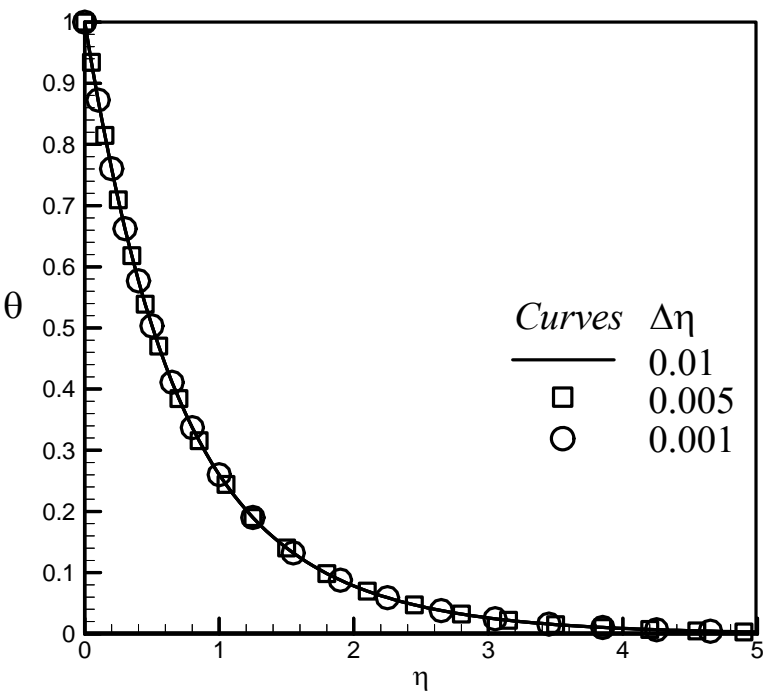

Figure 4. Distribution of temperature profiles for $\Delta \eta$.

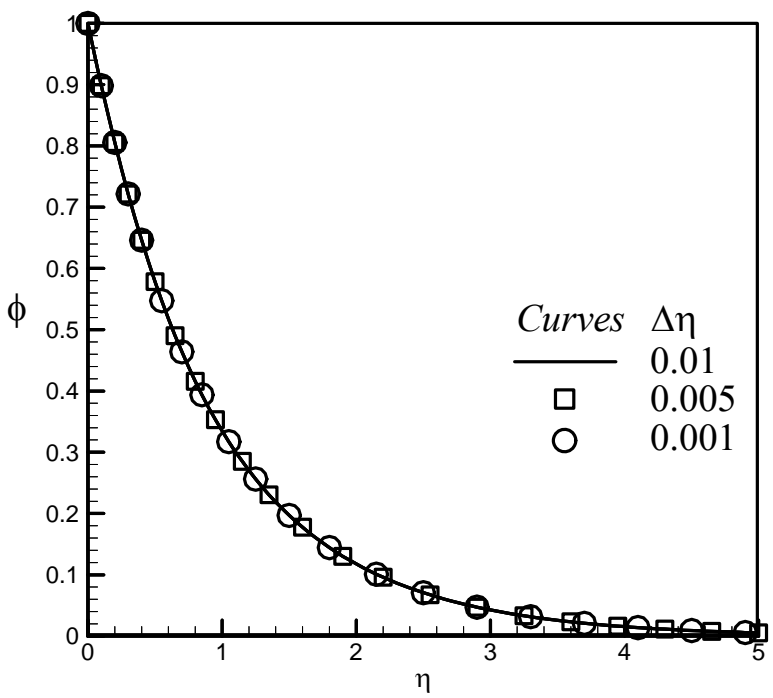

Figure 5. Distribution of concentration profiles for $\Delta \eta$.

\section{Results and Discussions}

For the purpose of discussing the results of the flow field represented in the Figure 1, the numerical calculations are presented in the form of non-dimensional velocity, microrotation, temperature and concentration profiles. The values of buoyancy parameter $G r$ is taken to be both positive to represent cooling of the plate. The parameters are chosen arbitrarily where $\operatorname{Pr}=0.71$ corresponds physically to air at $20^{\circ} \mathrm{C}, \operatorname{Pr}=1.0$ corresponds to the electrolyte solution such as salt water and $\operatorname{Pr}=7.0$ corresponds to water, and $S c=0.22,0.6$ and 1.0 corresponds to hydrogen, water vapor and methanol respectively at $25^{\circ} \mathrm{C}$ and 1 atmosphere. The values of Dufour and Soret numbers are chosen in such a way their production is constant provided that the meat temperature $T_{m}$ is kept constant as well.
Due to free convection problem positive large values of $G r=10$ is chosen. The value of $M=0.5, D a=1.0$, $S c=0.5, \operatorname{Pr}=0.71$ and $\xi=0.5$. However, numerical computations have been carried out for different values of the vortex viscosity parameter $(\Delta)$, surface nonlinearity parameter $(n)$, Eckert number $(E c)$, constant parameter $(\gamma)$, Dufour number $(D u)$, Soret number $(S r)$ and suction parameter $\left(f_{w}\right)$. The numerical results for the velocity, microrotation, temperature and concentration profiles are displayed in Figures 6-33.

Figure 6 shows the effect of vortex viscosity parameter $\Delta(\Delta=0.2,0.5,1,1.5$ are chosen $)$ on the velocity profiles. From here we see that velocity profiles decrease with the increase of $\Delta$. Figure 7 demonstrates the effect of $\Delta$ on the microrotation profiles. From this figure it is seen that microrotation increases very evidently with the increase of the vortex viscosity parameter $\Delta$. It is also understood that as the vortex viscosity increases the rotation of the micropolar constituents gets induced in most part of the boundary layer where kinematic viscosity dominates the flow. From Figure 8 it is found that the temperature profiles increase for the increase of $\Delta$. The effect of vortex viscosity parameter $\Delta$ on the concentration profile is not so noteworthy displayed in Figure 9.

The effects of the surface nonlinearity constant $n$ are characterized in the Figures 10-13. At the beginning the velocity profiles decrease with the increase of the value of $n(n=1,2,3,4)$ but far away from the plate they increase after $\eta=1.76$ displayed in Figure 10. Figure 11 expresses that the microrotation profiles at the beginning increase extensively but at a distance from the plate they overlap and start to decrease very slowly. Figures 12 and 13 enlighten the temperature and the concentration profiles for the increasing influence of the parameter $n$ respectively.

Figures 14-17 exhibit the velocity, microrotation, temperature and concentration profiles for the different values of the Eckert number $E c(0.03,0.1,0.5$ and 1.0). Figure 14 demonstrates that the effect of the $E c$ on velocity profiles very significant. We observe that velocity increases rapidly with increasing the value of $E c$. From the Figure 15 we notice that microrotation profiles decreases with the increase of the value of $E c$. Figure 16 presents the increasing effect of $E c$ on the temperature profiles. The concentration profiles decrease with the increase of the value of $E c$ illustrated in Figure 17.

Figures 18-21 represent the influence of the constant parameter $\gamma$ for the values $\gamma=0,1,2,5$. All the profiles except microrotation profiles decrease with the increase of $\gamma$. The effects of $\gamma$ are very significant smooth on the distributions. The microrotation profiles increase with the increase of the value of $\gamma$.

It is observed from the Figure 22 that with the increase 


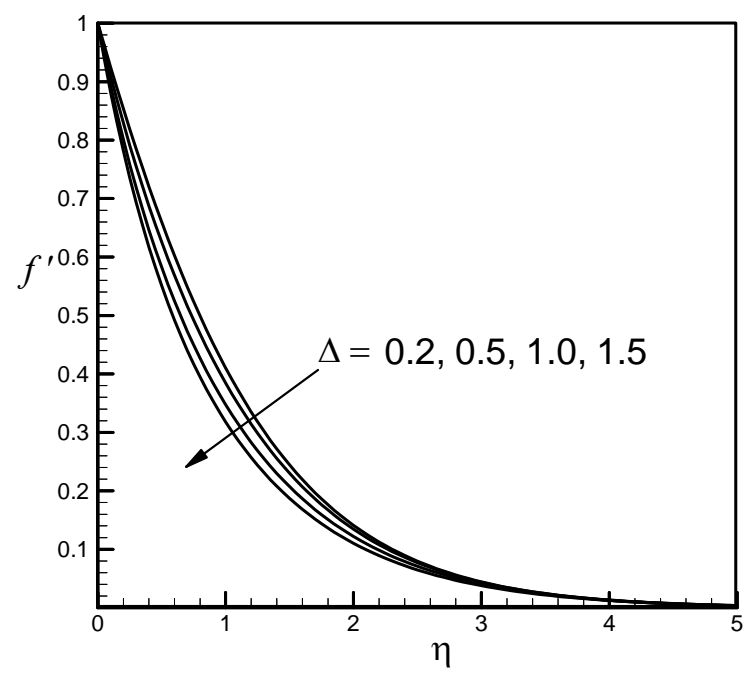

Figure 6. Distribution of velocity profiles for $\Delta$.

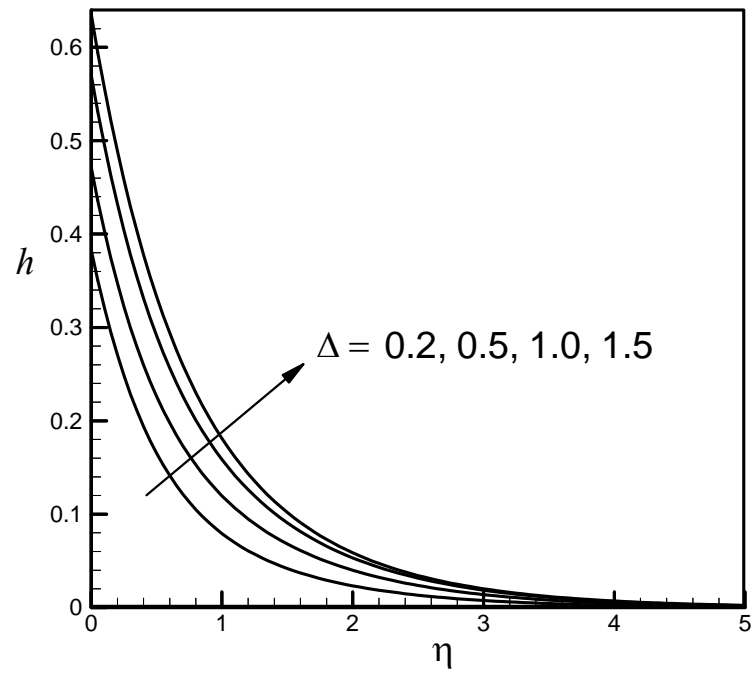

Figure 7. Distribution of microrotation profiles for $\Delta$.

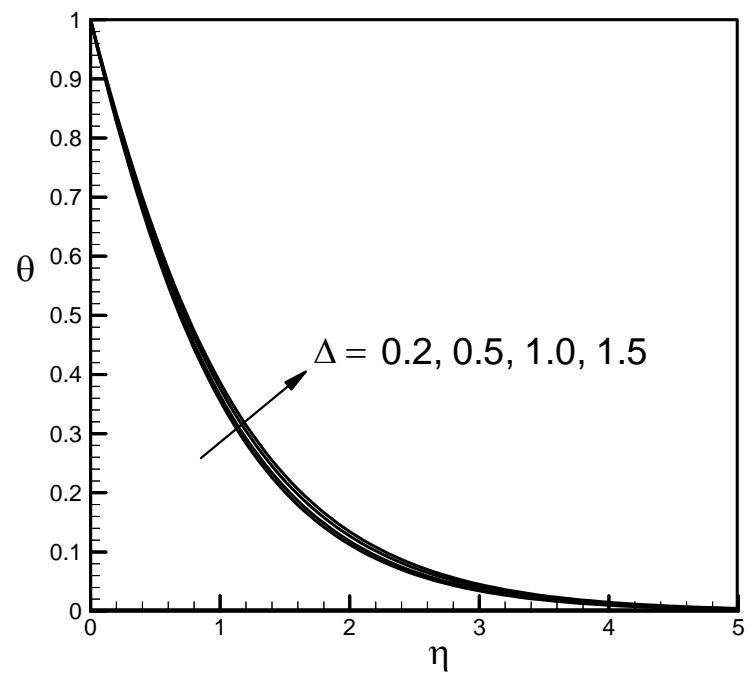

Figure 8. Distribution of temperature profiles for $\Delta$.

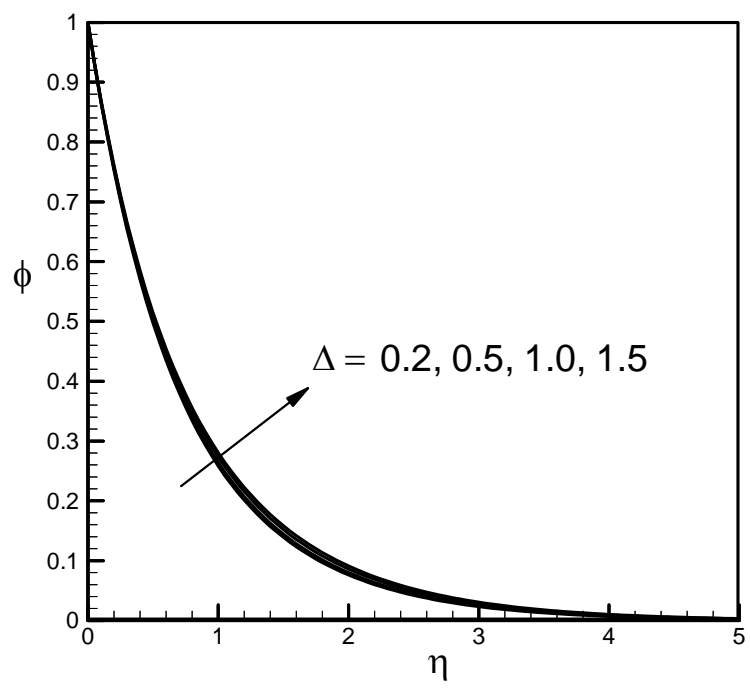

Figure 9. Distribution of concentration profiles for $\Delta$.

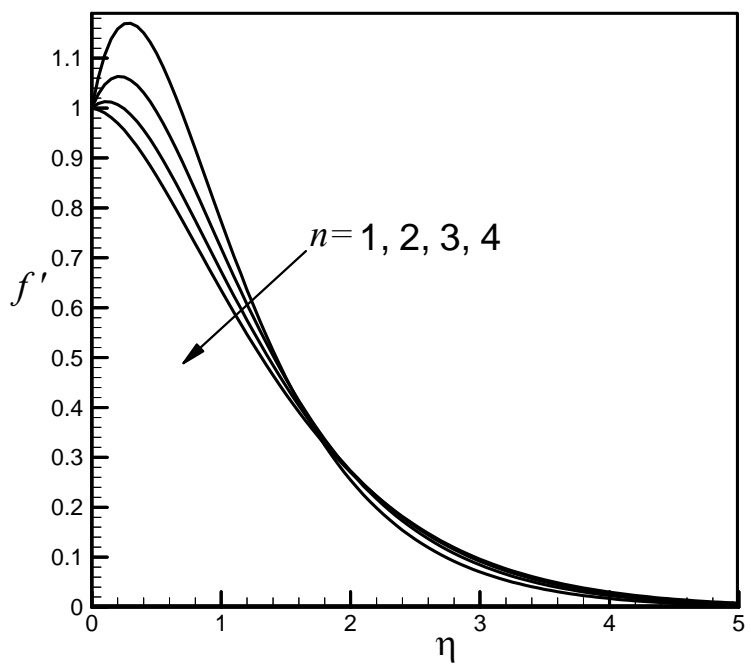

Figure 10. Distribution of velocity profiles for $\boldsymbol{n}$.

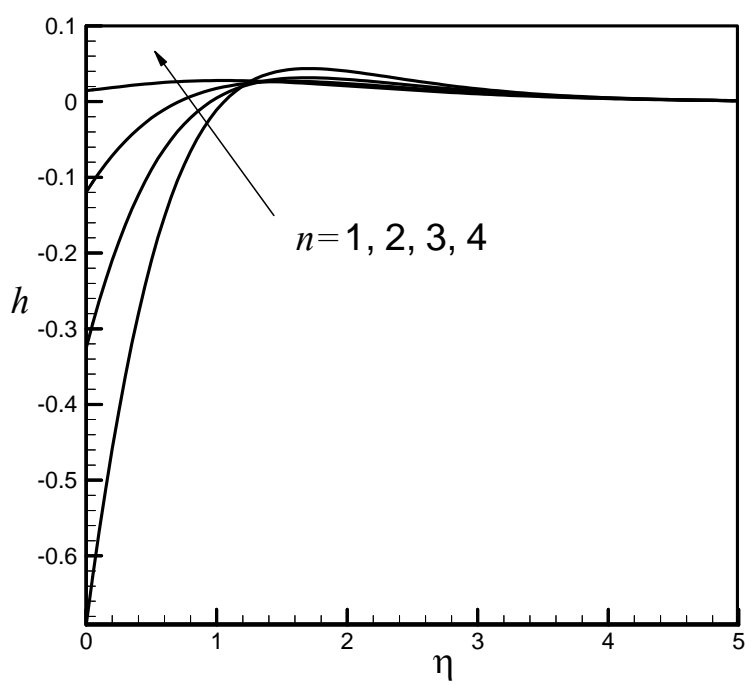

Figure 11. Distribution of microrotation profiles for $\boldsymbol{n}$. 


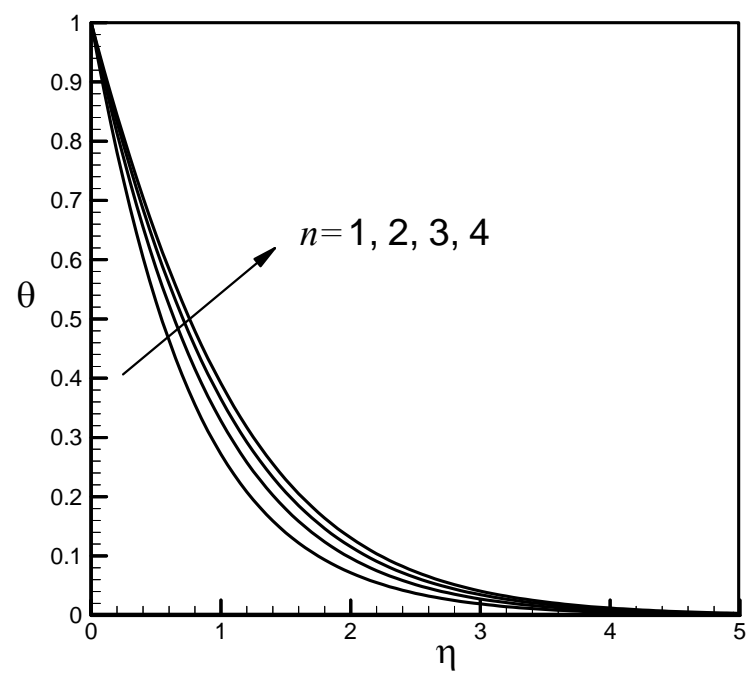

Figure 12. Distribution of temperature profiles for $\boldsymbol{n}$.

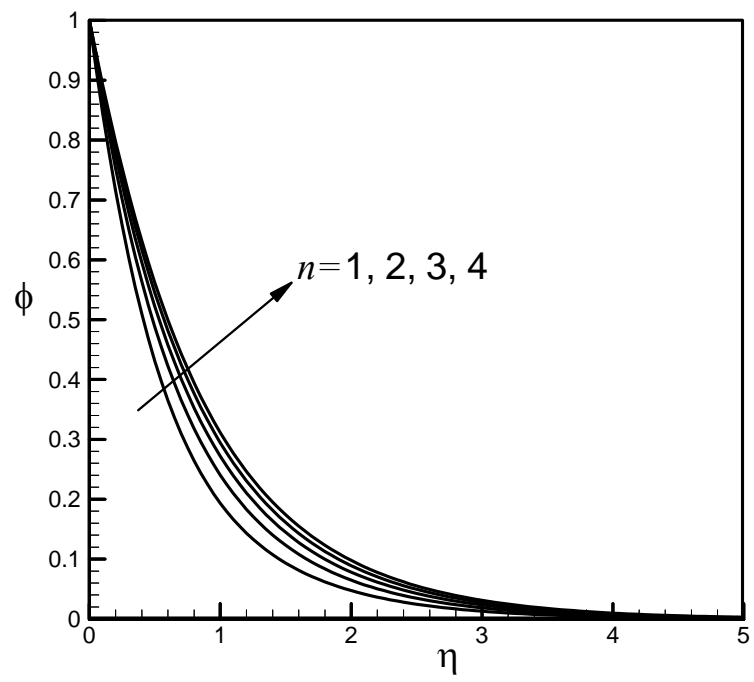

Figure 13. Distribution of concentration profiles for $\boldsymbol{n}$.

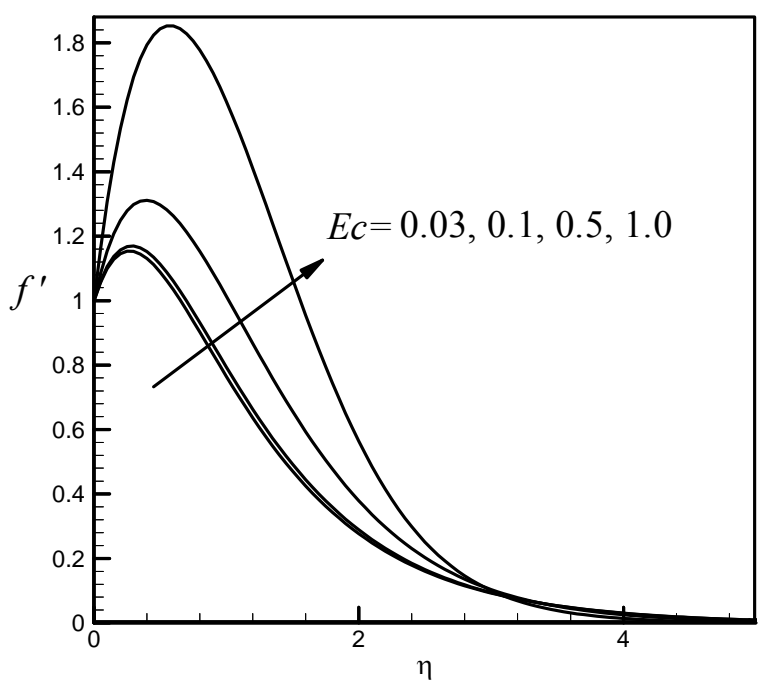

Figure 14. Distribution of velocity profiles for $E c$.

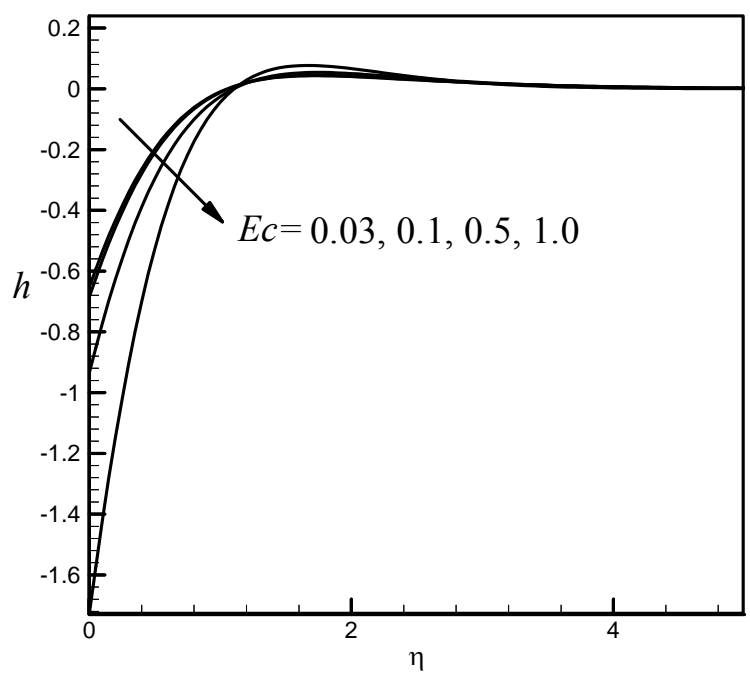

Figure 15. Distribution of microrotation profiles for $\boldsymbol{E} \boldsymbol{c}$.

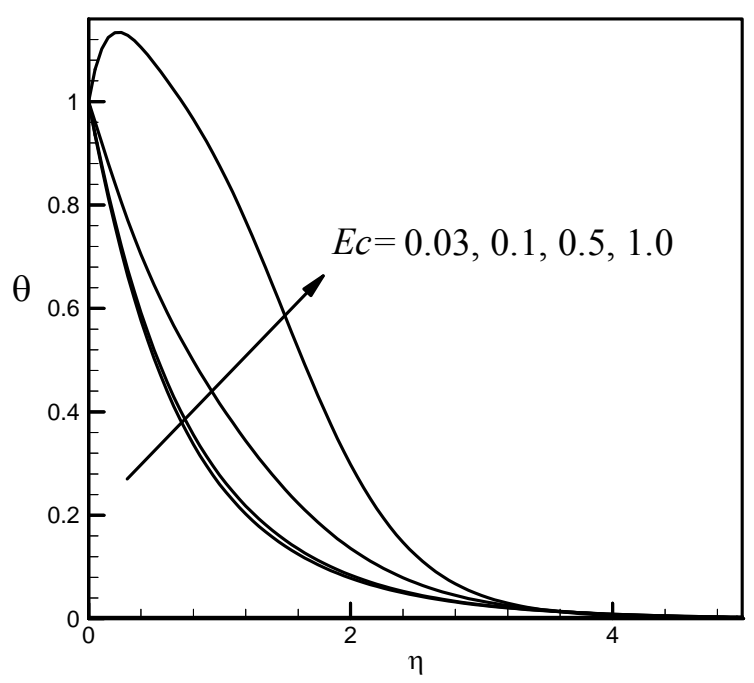

Figure 16. Distribution of temperature profiles for $E c$.

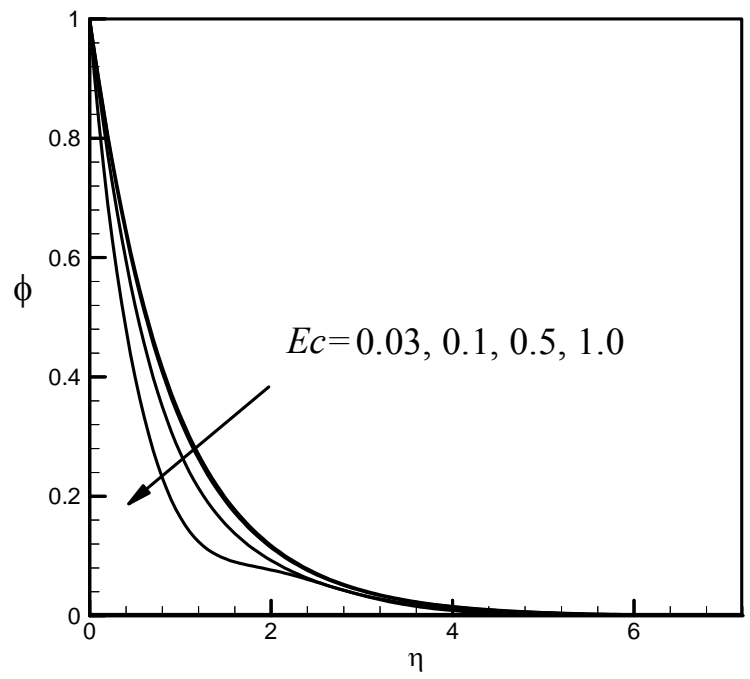

Figure 17. Distribution of concentration profiles for $\boldsymbol{E c}$. 


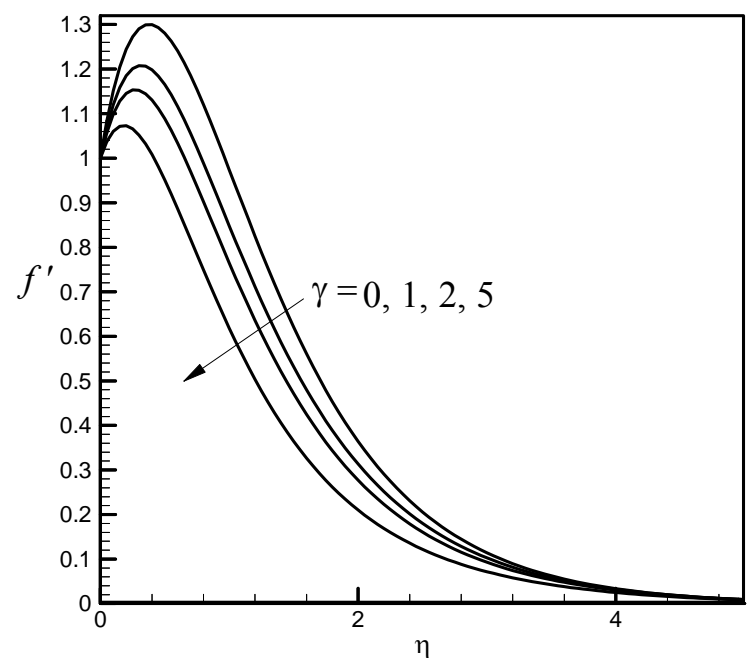

Figure 18. Distribution of velocity profiles for $\gamma$.

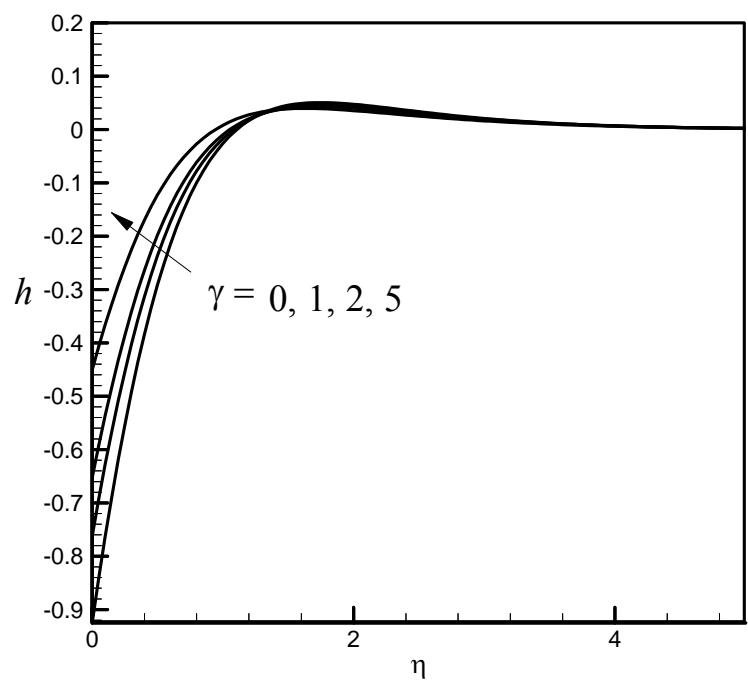

Figure 19. Distribution of microrotation profiles for $\gamma$.

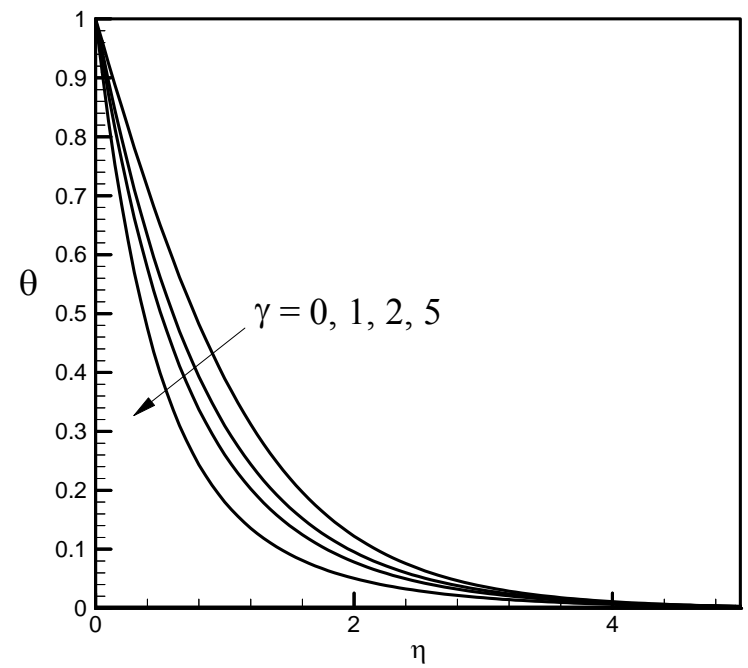

Figure 20. Distribution of temperature profiles for $\gamma$.

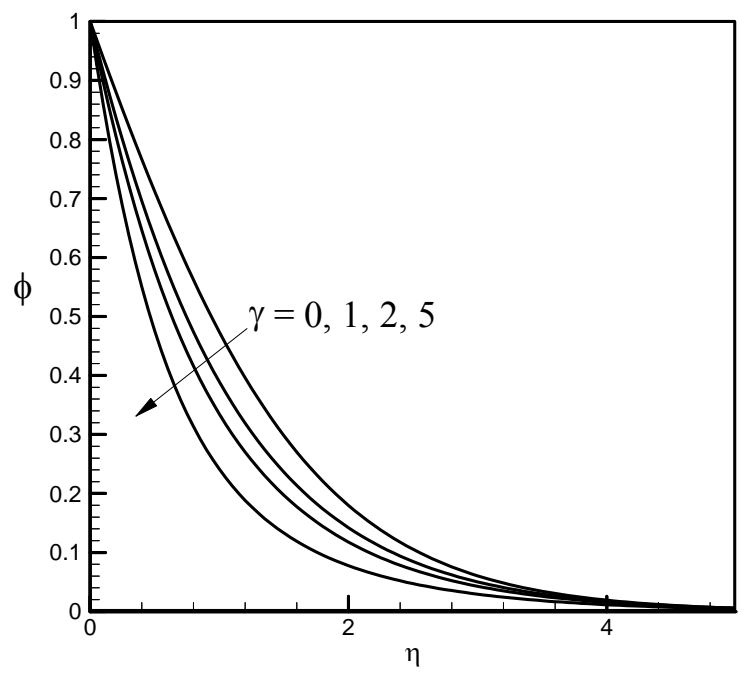

Figure 21. Distribution of concentration profiles for $\gamma$.

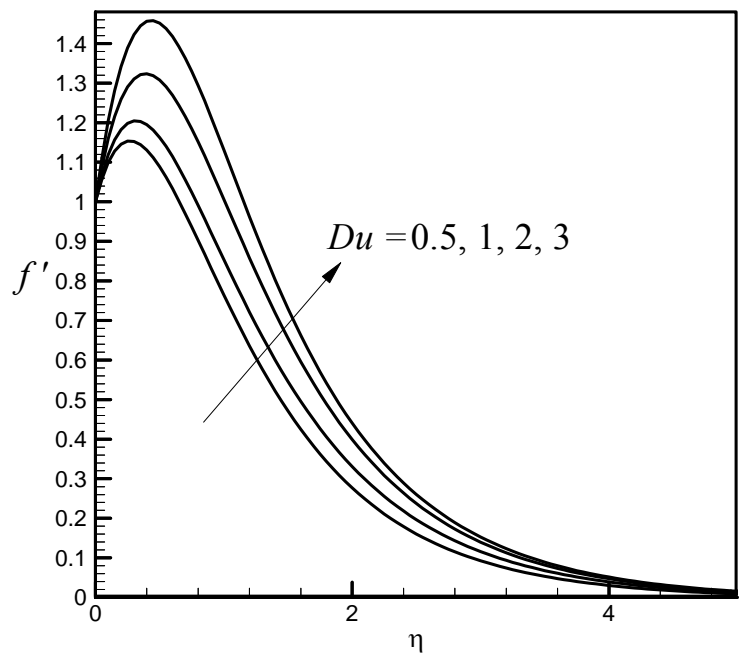

Figure 22. Distribution of velocity profiles for $\mathrm{Du}$.

of the value of $D u$ the velocity profiles occur higher. The effect of $D u$ on the microrotation profiles is insignificant illustrated in Figure 23. From Figure 24, it is noticed that $D u$ has remarkable effect on temperature profiles; quantitatively when $\eta=1.0 \mathrm{Du}$ increases from 0.5 to 1 and there is $23.08 \%$ increase in the temperature value, whereas the corresponding increase is $19.05 \%$, when $D u$ increases from 2 to 3 . The Dufour number has a falling effect on the concentration field shown in Figure 25. Quantitatively when $\eta=1.0$ and $D u$ increases from 0.5 to 1 , there is $15.56 \%$ decrease in the concentration value, whereas the corresponding decrease is $6.67 \%$ when $D u$ increases from 2 to 3 .

Figures 26-29 display the effects of the Soret number $S r$ on the velocity, microrotation, temperature and concentration profiles respectively. It is observed that $S r$ has very negligible effect on the velocity, microrotation and temperature profiles. Figure 29 reveals that the Soret 


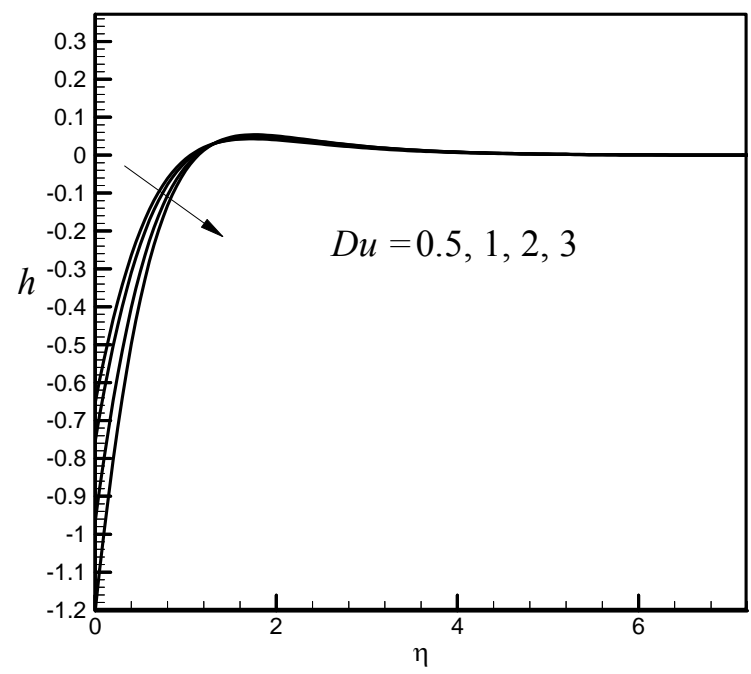

Figure 23. Distribution of microrotation profiles for $\mathrm{Du}$.

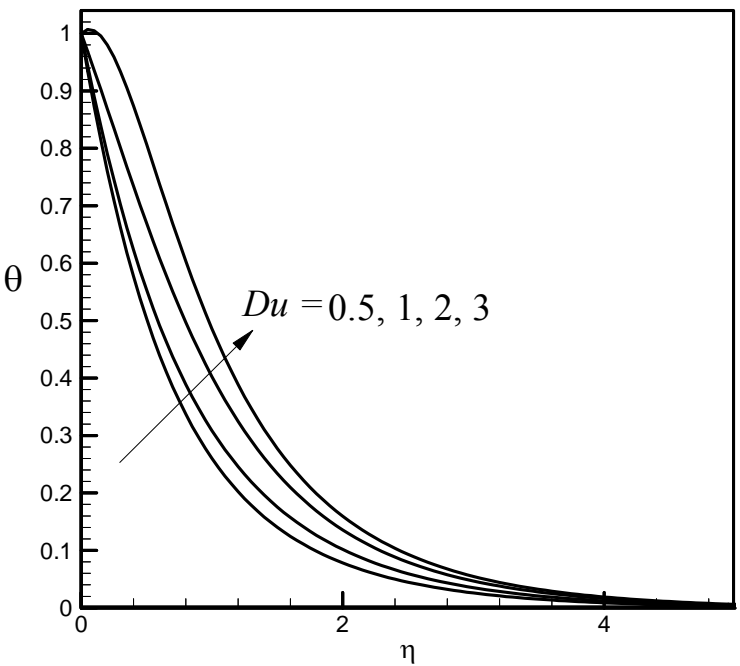

Figure 24. Distribution of temperature profiles for $D u$.

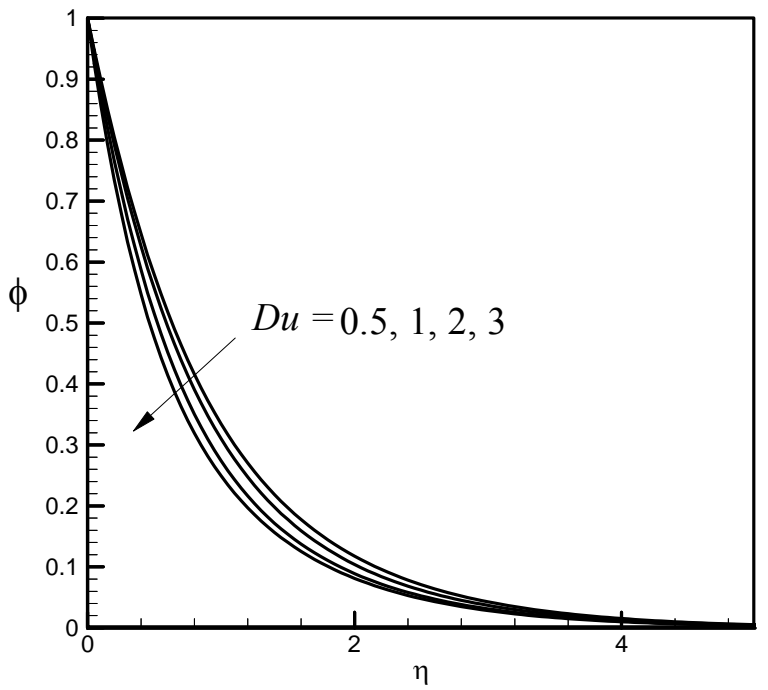

Figure 25. Distribution of concentration profiles for $\mathrm{Du}$.

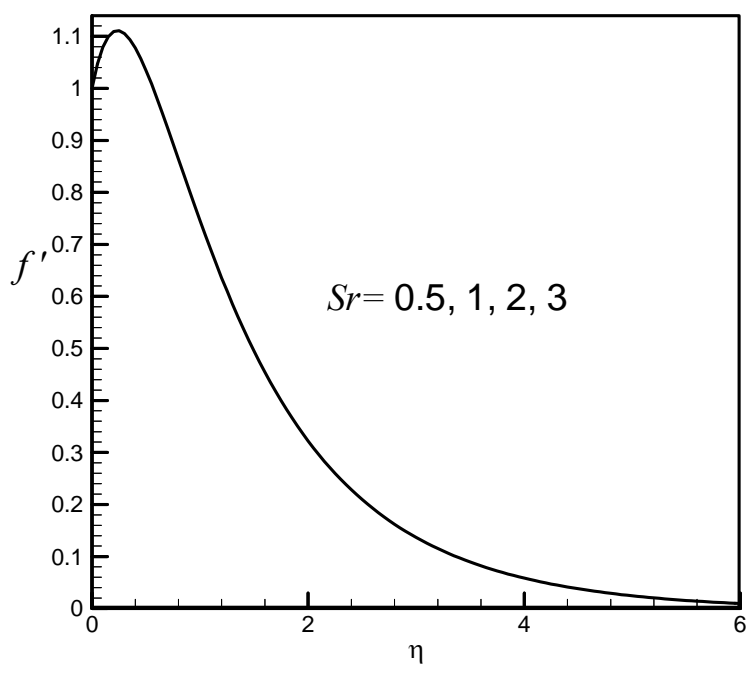

Figure 26. Distribution of velocity profiles for $S r$.

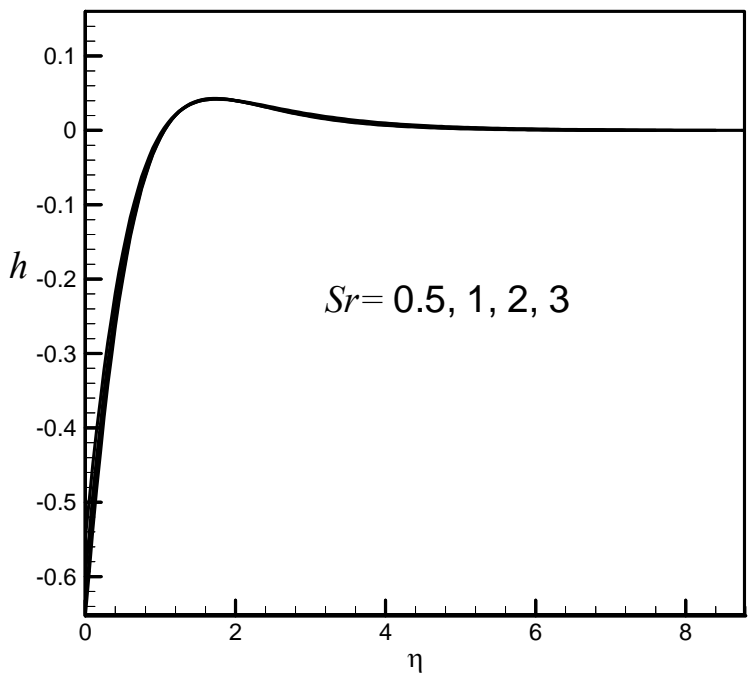

Figure 27. Distribution of microrotation profiles for $\boldsymbol{S r}$.

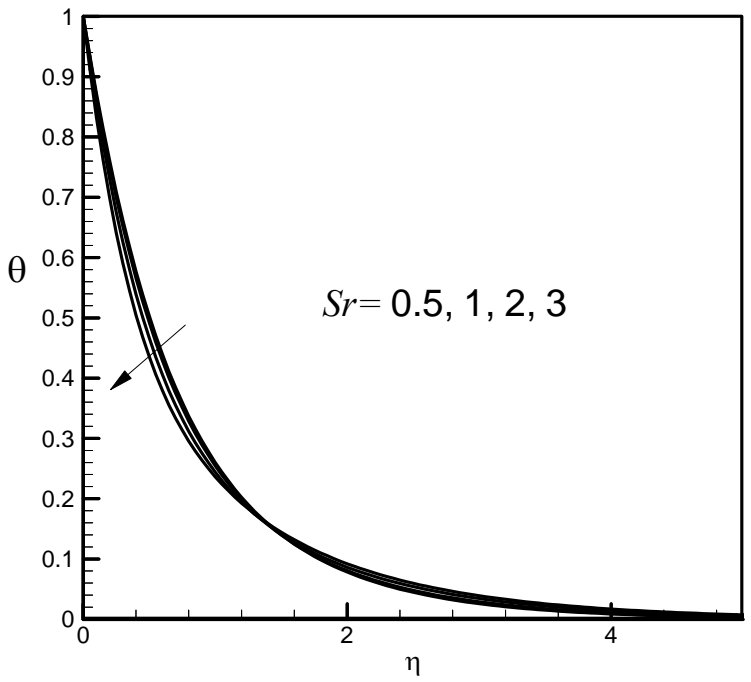

Figure 28. Distribution of temperature profiles for $\boldsymbol{S} r$. 
number $S r$ influences the concentration profiles to a great extent. Quantitatively when $\eta=1.0$ and $D u$ increases from 0.5 to 1 , there is $23.08 \%$ decrease in the concentration value, whereas the corresponding decrease is $23.07 \%$ when $D u$ increases from 2 to 3 .

Figure 30 displays that the suction parameter $f_{w}$ has strong effect on the velocity profiles. With the increase of the value of $f w$ the velocity profiles decrease. Elaborately when $\eta=1.0$ and $D u$ decreases from 0 to 0.5 , there is $176.79 \%$ decrease in the concentration value, whereas the corresponding decrease is $21.47 \%$ when $D u$ increases from 1 to 3 . It is observed that, when suction $f_{w}$ increases, the microrotation increase monotonically seen in Figure 31. These Figures 32 and 33 indicate that temperature as well as concentration profiles decrease with the increase of suction velocity or mass transfer parameter frequently.

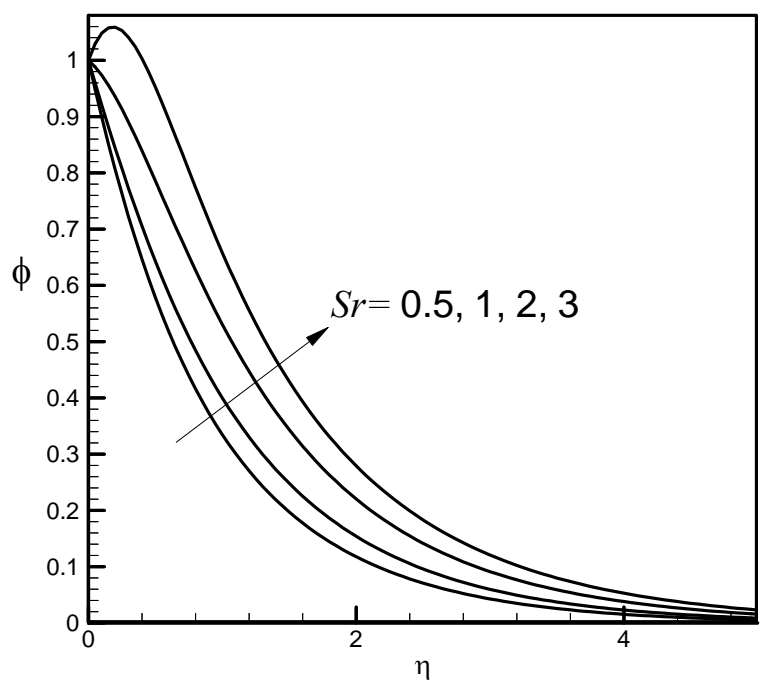

Figure 29. Distribution of concentration profiles for $S r$.

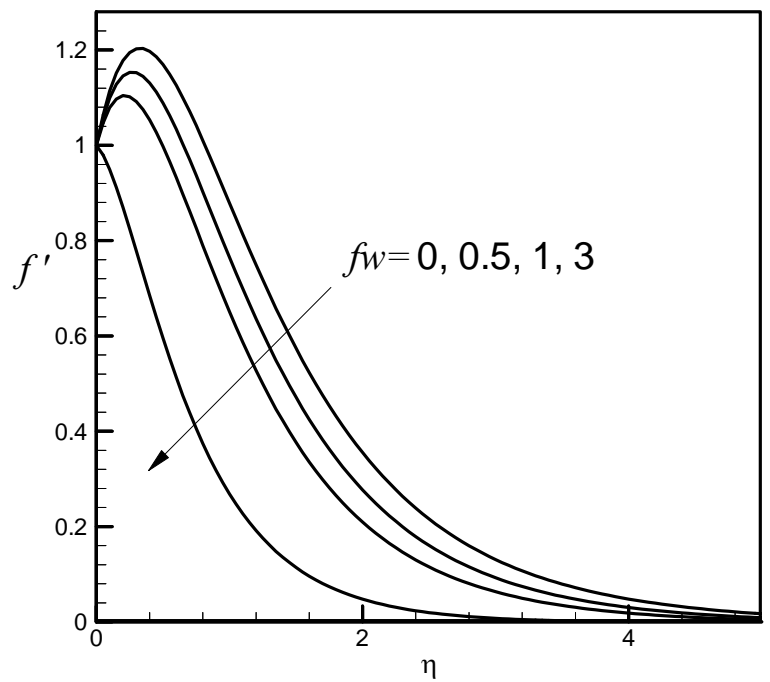

Figure 30. Distribution of velocity profiles for $f w$.

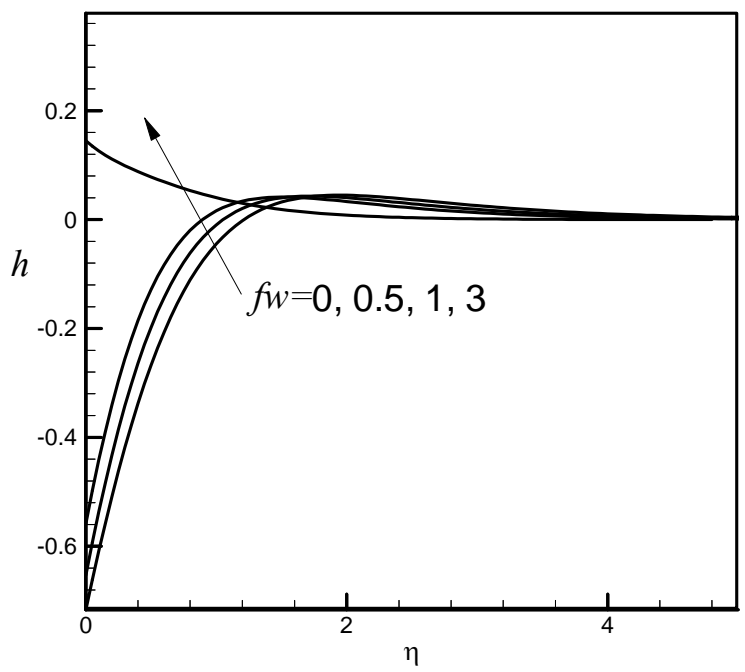

Figure 31. Distribution of microrotation profiles for $f w$.

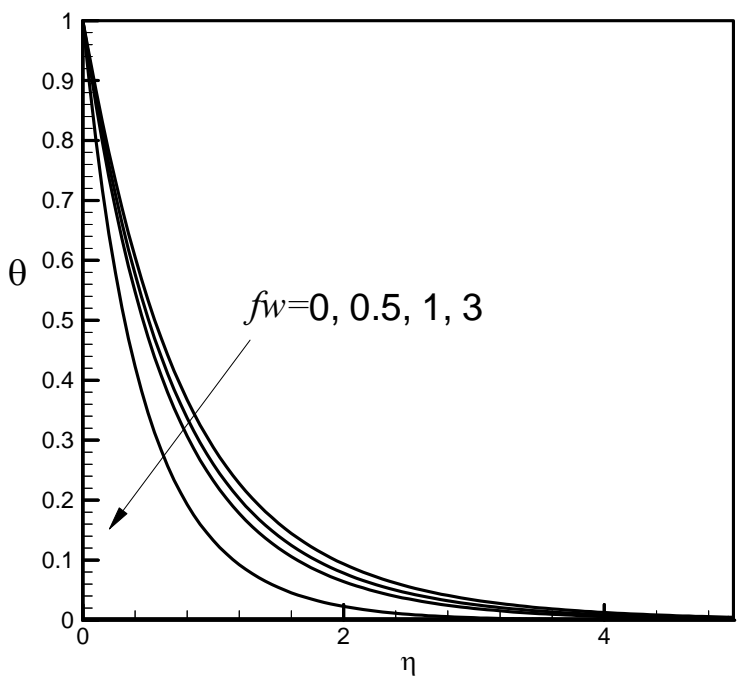

Figure 32. Distribution of temperature profiles for $f w$.

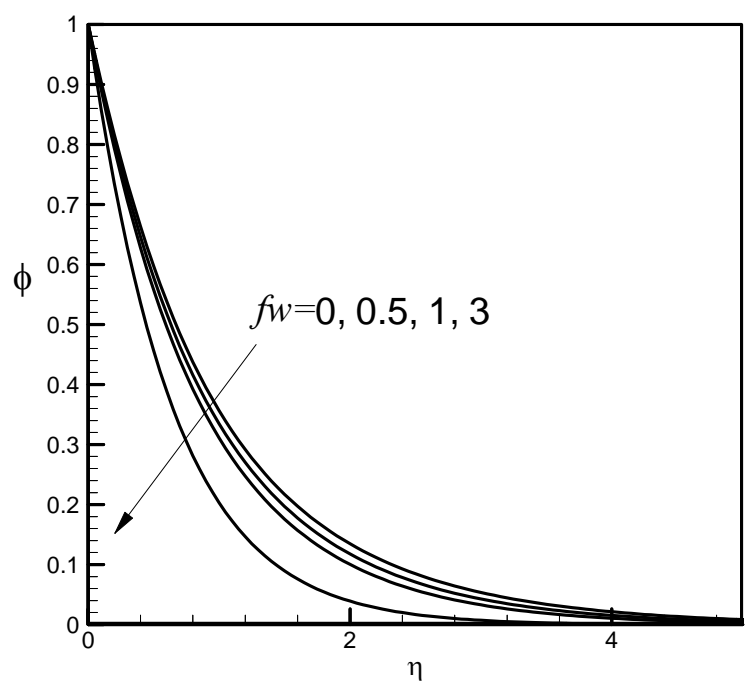

Figure 33. Distribution of concentration profiles for $f w$. 
Finally, Finally, the effects of various parameters on the skin friction $C_{f}$, couple stress $M_{w}$, local Nusselt number $N u$ and local Sherwood number $S h$ are shown in the Tables 1-2.

\section{Conclusions}

In the present paper, Soret Dufour effect on the boundary layer flow and heat transfer of microrotation fluid over a nonlinear stretching plate in the presence of suction has been studied. The governing momentum and energy equations were transformed to a set of non linear ordinary differential equations by employing the appropriate similarity transformations and solve numerically for various combinations of problem parameters. The effects of the vortex viscosity parameter $(\Delta)$, surface nonlinearity parameter $(n)$, Eckert number $(E c)$, constant parameter $(\gamma)$, Dufour number $(D u)$, Soret number $(S r)$ and suction parameter $(f w)$ are investigated through the use of graphs.

Table 1. $C_{f}, M w, N u$ and $S h$ for different values of the parameters $\Delta$ and $n$.

\begin{tabular}{ccccc}
\hline Parameters & $C_{f}$ & $M_{w}$ & $N u$ & $S h$ \\
\hline$\Delta=0.2$ & -0.7660054 & -0.6785573 & 0.9187514 & 1.4347764 \\
0.5 & -0.9431672 & -0.7418682 & 0.8969659 & 1.4211495 \\
1.0 & -1.1402750 & -0.8065383 & 0.8652346 & 1.4042243 \\
1.5 & -1.2705812 & -0.8432893 & 0.8377928 & 1.3924873 \\
$n=2.0$ & 0.6510607 & 0.6598670 & 1.0016252 & 1.4025156 \\
3.0 & 0.2393268 & 0.2751800 & 0.8948076 & 1.2650374 \\
4.0 & -0.0287619 & 0.0197969 & 0.8257597 & 1.1776228 \\
\hline
\end{tabular}

Table 2. $C_{f}, M w, N u$ and $S h$ for different values of the parameter $E c, \gamma, D u, S r$ and $f_{w}$.

\begin{tabular}{ccccc}
\hline Parameters & $C_{f}$ & $M_{w}$ & $N u$ & $S h$ \\
\hline$E c=0.10$ & 1.3717933 & 1.2810219 & 1.2871015 & 1.0869111 \\
0.50 & 1.8711019 & 1.7189631 & 0.7748798 & 1.2717607 \\
1.00 & 3.4607307 & 3.2494880 & -1.5177258 & 1.9183527 \\
$\gamma=0.0$ & 1.8501324 & 1.7020239 & 0.7579262 & 0.6120285 \\
1.0 & 1.5247058 & 1.4135722 & 1.0978664 & 0.8674080 \\
5.0 & 0.9011038 & 0.8872078 & 1.8941767 & 1.4905104 \\
$D u=1$ & 1.5078442 & 1.3967581 & 1.1508341 & 1.1396871 \\
2 & 1.9322204 & 1.7744343 & 0.6155264 & 1.3127209 \\
3 & 2.4036488 & 2.2140699 & -0.2279146 & 1.5484008 \\
$S r=1$ & 1.2721836 & 1.1945495 & 1.4130040 & 0.8184983 \\
2 & 1.1943642 & 1.1250180 & 1.5801953 & 0.2060201 \\
3 & 1.0959358 & 1.0378509 & 1.8467657 & -0.7052097 \\
$f w=0$ & 1.4328645 & 1.1173236 & 1.2121609 & 0.9927766 \\
1 & 1.1166248 & 1.2559658 & 1.5013565 & 1.1358380 \\
3 & -0.2894534 & -0.2193329 & 2.2488293 & 1.5064022 \\
\hline
\end{tabular}

From the present study the following conclusions are made:

1) The effect of vortex viscosity $(\Delta)$ parameter on velocity and microrotation is prominent;

2) Nonlinearity of the stretching surface $(n)$ is effective on the boundary layer flow;

3) Effect of Eckert number $(E c)$ is uniform;

4) Boundary layer growth can be controlled by using constant parameter $(\gamma)$;

5) The Dufour $(D u)$ effect is significant;

6) The Soret number $(S r)$ plays a role on concentration;

7) The effect of suction parameter $(f w)$ is dominating on the velocity, microrotation, temperature and concentration profiles. So, using suction boundary layer growth can be stabilized.

\section{REFERENCES}

[1] S. Ostrach, "An Analysis of Laminar Free-Convection Flow and Heat Transfer about a Flat Plate Parallel to the Direction of the Generating Body Force," Technical Note, nACA Report, Washington DC, 1952. http://naca.central.cranfield.ac.uk/report.php?NID $=5021$

[2] R. M. Goody, "The Influence of Radiative Transfer on Cellular Convection," Journal of Fluid Mechanics, Vol. 1, No. 4, 1956, pp. 424-435. doi:10.1017/S0022112056000263

[3] B. C. Sakiadis, "Boundary-Layer Behavior on Continuous Solid Surfaces: I. Boundary-Layer Equations for Two-Dimensional and Axisymmetric Flow," AIChE Journal, Vol. 7, No. 1, 1961, pp. 26-28. doi:10.1002/aic.690070108

[4] A. C. Eringen, "Theory of Micropolar Fluids," Journal of Mathematics and Mechanics, Vol. 16, No. 1, 1966, pp. $1-18$.

[5] L. J. Crane, "Flow past a Stretching Sheet," Zeitschrift für Angewandte Mathematik und Physik (ZAMP), Vol. 21, No. 4, 1970, pp. 645-647. doi:10.1007/BF01587695

[6] E. M. Sparrow, "Radiation Heat Transfer," Augmented Edition, Hemisphere Publishing Corp., Washington DC, 1978.

[7] O. Aydin and I. Pop, "Natural Convection from a Discrete Heater in Enclosures Filled with a Micropolar Fluid," International Journal of Engineering Science, Vol. 43, No. 19-20, 2005, pp. 1409-1418. doi:10.1016/j.ijengsci.2005.06.005

[8] M.-I. Char and C.-L. Chang, "Effect of Wall Conduction on Natural Convection Flow of Micropolar Fluids along a Flat Plate," International Journal of Heat and Mass Transfer, Vol. 40, No. 15, 1997, pp. 3641-3652. doi:10.1016/S0017-9310(97)00006-9

[9] H. A. M. El-Arabawy, "Effect of Suction/Injection on the Flow of a Micropolar Fluid past a Continuously Moving Plate in the Presence of Radiation," International Journal of Heat and Mass Transfer, Vol. 46, No. 8, 2003, pp. 1471-1477. doi:10.1016/S0017-9310(02)00320-4 
[10] A. Ishak, R. Nazar and I. Pop, "The Schneider Problem for a Micropolar Fluid," Fluid Dynamics Research, Vol. 38, No. 7, 2006, pp. 489-502. doi:10.1016/j.fluiddyn.2006.03.004

[11] M. E. Karim, M. A. Samad and M. A. Sattar, "Steady MHD Free Convection Flow with Thermal Radiation past a Vertical Porous Plate Immersed in a Porous Medium," Research Journal of Mathematics and Statistics, Vol. 3, No. 4, 2011, pp. 141-147. http://www.maxwellsci.com/print/rjms/v3-141-147.pdf

[12] Y. Y. Lok, N. Amin and I. Pop, "Unsteady Boundary Layer Flow of a Micropolar Fluid near the Rear Stagnation Point of a Plane Surface," International Journal of Thermal Sciences, Vol. 42, No. 11, 2003, pp. 995-1001. doi:10.1016/S1290-0729(03)00079-6

[13] R. Nazar, A. Ishak and I. Pop, "Unsteady Boundary Layer Flow over a Stretching Sheet in a Micropolar Fluid," International Journal of Mathematical, Physical and Engineering Sciences, Vol. 2, No. 3, 2008, pp. 161-165.

[14] M. M. Rahman, M. A. Rahman, M. A. Samad and M. S. Alam, "Heat Transfer in a Micropolar Fluid along a NonLinear Stretching Sheet with a Temperature-Dependent Viscosity and Variable Surface Temperature," International Journal of Thermophysics, Vol. 30, No. 5, 2009, pp. 1649-1670.

[15] M. M. Rahman, M. J. Uddin and A. Aziz, "Convective Flow of Micropolar Fluid in a Porous Medium with Variable Electric Conductivity, Surface Heat Flux and NonUniform Heat Source (or Sink)," International Journal of Energy \& Technology, Vol. 25, No. 2, 2010, pp. 1-18.

[16] H. S. Takhar, R. Bhargava, R. S. Agrawal and A. V. S. Balaji, "Finite Element Solution of a Micropolar Fluid Flow and Heat Transfer between Two Porous Disks," International Journal of Engineering Science, Vol. 38, No.
17, 2000, pp. 1907-1922. doi:10.1016/S0020-7225(00)00019-7

[17] E. R. G. Eckert and R. M. Drake, "Analysis of Heat and Mass Transfer," McGraw-Hill, New York, 1972.

[18] T. Hayat and F. A. Hendi, "Thermal-Diffusion and Diffusion-Thermo Effects on MHD Three-Dimensional Axisymmetric Flow with Hall and Ion-Slip Currents," Journal of American Science, Vol. 8, No. 1, 2012, pp. 284-294. http://www.jofamericanscience.org/journals/am-sci/am08 01/042_7833am0801_284_294.pdf

[19] O. D. Makinde and P. O. Olanrewaju, "Unsteady Mixed Convection with Soret and Dufour Effects past a Porous Plate Moving through a Binary Mixture of Chemical Reacting Fluid," Chemical Engineering Communications, Vol. 198, No. 7, 2011, pp. 920-938. doi:10.1080/00986445.2011.545296

[20] S. Shateyi, S. S. Motsa and P. Sibanda, "The Effects of Thermal Radiation, Hall Currents, Soret, and Dufour on MHD Flow by Mixed Convection over a Vertical Surface in Porous Media," Mathematical Problems in Engineering, Vol. 2010, No. 1, 2010, pp. 1-20. doi: $10.1155 / 2010 / 627475$

[21] D. Srinivasacharya and K. Kaladhar, "Mixed Convection in a Couple Stress Fluid with Soret and Dufour Effects," Internatioanl Journal of Applied Mathematics and Mechanics, Vol. 7, No. 20, 2011, pp. 59-71.

[22] P. R. Nachtsheim and P. Swigert, "Satisfaction of the Asymptotic Boundary Conditions in Numerical Solution of the Systems of Non-Linear Equations of Boundary Layer Type," Ph.D. Thesis, NASA TN D-3004, Washington DC, 1965.

http://ntrs.nasa.gov/archive/nasa/casi.ntrs.nasa.gov/19650 $026350 \_1965026350 . p d f$ 